\title{
The Prevalence and Factors Associated with Mental Distress among College Students in Southern Ethiopia: A Cross-Sectional Study
}

\author{
Tesfalem Teshome Tessema ${ }^{1}$, Temesgen Abdi Gebremariam ${ }^{1}$, Etaferahu \\ Alemayehu Abebe ${ }^{2}$, Ribka Dinku Gebre ${ }^{1}$
}

OPEN ACCESS

Citation: Tesfalem Teshome Tessema, Temesgen Abdi Gebremariam, Etaferahu Alemayehu Abebe, Ribka Dinku Gebre. The Prevalence and Factors Associated with Mental Distress among College Students in Southern Ethiopia : A CrossSectional Study

Ethiop J Health Sci. 2019;29(3):353.

doi:http://dx.doi.org/10.4314/ ejhs.v29i3.7

Received: October 27, 2018

Accepted: January 21, 2019

Published: May 1, 2019

Copyright: (C) 2019 Tesfalem Teshome Tessema, et al. This is an open access article distributed under the terms of the Creative Commons Attribution License, which permits unrestricted use, distribution, and reproduction in any medium, provided the original author and source are credited.

Funding: Hawassa College of Health Sciences

Competing Interests: The authors declare that this manuscript was approved by all authors in its form and that no competing interest exists.

Affiliation and Correspondence:

${ }^{1}$ Department of Public Health, Hawassa College of Health Sciences, Hawassa Ethiopia

${ }^{2}$ Southern Regional State Health Office, Hawassa Ethiopia

*Email: tesfabemnet2005@gmail.com

\author{
ABSTRACT
}

BACKGROUND: Although mental health problems affect the general population, students are highly vulnerable to psychological disorders. Mental distress among students is a cause of concern as it may impair their behavior and diminish learning, which could negatively affect their academic performance. Therefore, the objective of this study was to assess the prevalence and the factors associated with mental distress among college students in Southern Ethiopia.

METHODS: Institution based cross-sectional study was conducted among 280 regular students of Hawassa College of Health Sciences. Mental distress was measured using a Self-Reporting Questionnaire (SRQ-20). The data were entered into Epi-Info version 3.5.4 and analyzed using Statistical Package for Social Science (SPSS) version 20. Multivariable logistic analysis was performed following bivariate analysis to adjust for the effect of confounders.

RESULT: The prevalence of mental distress among the study subjects was found to be 59 (22.30\%). Female students were 2.08 times more likely to have mental distress as compared to male students (AOR $=2.08$ [95\% CI: 1.14-3.82]). The odds of mental distress among third-year students was 60\% lower compared to second-year students $[A O R=0.40 ; 95 \% C I,(0.19,0.87)]$. Students who were from Amhara ethnic group were 3.37 times more likely to have mental distress as compared to students from Sidama ethnic group [AOR=3.37;95\%CI (1.13-10.09)]. Moreover, students who had Grade Point Average of below 60\% were 4.69 times more likely to be mentally distressed as compared to those students who scored Grade Point Average of greater or equal to 60\% $(A O R=4.69 ; 95 \%$ CI,[ 2.46, 8.96).

CONCLUSION: The finding suggests the importance of providing mental health counseling support for students as part of the teaching and learning process. More research to further explore the multiple factors influencing students' mental health condition should also be conducted.

KEYWORDS: Mental Distress, Self-Reporting Questionnaire (SRQ-20), Colleges Students, Southern Ethiopia 


\section{INTRODUCTION}

According to the World Health Organization (WHO), positive mental health is a state of wellbeing in which an individual can realize his/her own abilities, can interact positively with others, can cope with the stressors of life, can study and work productively and fruitfully, and is able to contribute to his/her family and community (1). Globally, an estimated 450 million people suffer from mental or behavioural disorders that is responsible for $33 \%$ of the years lived with disability (YLD) (2). In Ethiopia, mental illness is the leading non-communicable disorder contributing to $11 \%$ of the total burden of diseases, with schizophrenia and depression included in the top ten most burdensome conditions plus out-ranking HIV/AIDS (3).

Although mental health problems affect society as a whole, university students are found to have a high vulnerability for psychological disorders. This is due to the fact that university students have to face multiple stressors such as academic demands, workload and pressure to succeed, teacher and parents' pressure, financial burden and worry about the future. These stressors put students at an increased risk to develop a psychological problem. A high prevalence of mental distress among students is a cause of concern as it may impair behaviour of students, diminish learning which could negatively affect their academic performance, and ultimately affect patient care after their graduation (4-5).

Studies conducted globally to assess the level of mental distress among college students have reported the prevalence ranging from $17.70 \%$ to $41.1 \%$ (5-7). Cross-sectional studies conducted among students of Ethiopian higher academic institutions revealed the prevalence of mental distress with a wide variation of $21.6 \%$ in Adama University (8), 29\% in Mizan Aman Health Science College (9), 40.9 in the University of Gondar (10) and $30 \%$ and $49.1 \%$ in Hawassa University $(11,12)$.

The risk factors identified to increase the risk of mental distress among college/university students include financial difficulties, not having a satisfying relationship with the family, poor prospect of finding a job, emotional breakdown, substance abuse, suicide thought, suicide attempt, lack of interest in the discipline pursued, family history of mental illness, frequent conflicts with fellows and not attending religious programs (68). Furthermore, perceiving medical school as very stressful, and living environment as very crowded and having a feeling of insecurity about one's safety, lack of interest towards their field of study, not having close friends, lower grade than anticipated, lack of vacation or break and low social support were significantly associated with mental distress (10-12).

Most of the studies conducted in Ethiopia to assess the levels and risk factors for mental distress focused on university students. There are only a few studies documenting the prevalence of mental distress among mid-level medical students receiving training in regional-based medical colleges of Ethiopia. However, unlike university students, mid-level medical students have not been benefiting from the dormitory and cafeteria services in their college years. They are given very little financial support from the college (only 300 birr per month) and take responsibility to manage their academic and non-academic issues of life, such as residence and food. They usually rent homes which may be far from the college compound and live in groups. The students are also responsible to cover their food, stationery and related costs as well. We believe that these and other related factors make them more vulnerable for mental distress. Furthermore, they have been receiving very little research attention. Therefore, this study was conducted to assess the prevalence and factors associated with mental distress among mid-level medical students in Southern Ethiopia.

\section{MATERIALS AND METHODS}

Study setting and population: This study was conducted in Hawassa, the capital city of Southern Nation, Nationalities and Peoples Region (SNNPR) located $273 \mathrm{~km}$ South of Addis Ababa. According to the 2014 Central Statistical Agency (CSA) population projection, the city administration has a total population of 399,461 ,

DOI: http://dx.doi.org/10.4314/ejhs.v29i3.7 
out of which 199,768 were males and 199,693 females (13). Hawassa College of Health Sciences is one of the four public medical colleges established under Southern Regional Health Bureau. The college has been providing mid-level health professionals training for 1,300 regular students in nine different departments. The study population was second and third-year regular students of the college during the 2014/2015 academic year. First year students were excluded from the study considering the lack of their last year Grade Point Average (GPA) to assess the academic performance.

Study design, sample size and sampling techniques: An institution based cross-sectional study was conducted from January 1 to $30 / 2015$ among regular students of Hawassa College of Health Sciences. The sample size was determined using the formula of sample size determination for a single population proportion taking the proportion of mental distress among Adama University students $21 \%$ (8). Further assumptions considered include $95 \%$ level of confidence, $5 \%$ margin of error and $10 \%$ non-response rate. Accordingly, 280 students were required for the study. The calculated sample size was allocated to each of the departments and the years of the study based on their respective size. For each of the class level, the list of the student received from the college registrar was used as a sampling frame. Simple random sampling technique was then used to select study subjects from the registration list using the lottery method.

Method of data collection: Data on sociodemographic issues and the habits of substance use were collected using pre-tested and selfadministered questionnaire. The last year GPAs of the students were collected from college registrar. Unlike Ethiopian universities, the grading system of the mid-level medical students is calculated as a percentage. Therefore, the study subjects were categorized based on their first year academic achievement into two independent categories as those achieving below $60 \%$ or above/equal to $60 \%$.

Mental distress was measured using SelfReporting Questionnaire (SRQ-20) that was designed by the World Health Organization
(WHO) as an instrument to screen mental health disorder and found to be a reliable tool for use in different countries and cultures was used to measure the prevalence of mental distress (14). The tool was used in a number of previous studies to measure mental distress among university students $(8,10-12)$ and at the community-based studies as well $(15,16)$. The questionnaire consists of 20 items that are related to common symptoms of depression, anxiety and somatic complaints (such as sleep problems, headaches, and digestive problems). The study subjects were first asked to tick any symptoms that they had experienced in the past 30 days. Second, for every 'Yes' answer, the study subjects were asked to tick whether they had experienced that problem when they were not using alcohol or other drugs.

The English version of the questionnaire was used in this study considering the fact that the medium of education in higher academic institutions of Ethiopia including Hawassa College of Health Sciences is English. In this study, students who were found to have eight or more symptoms from the 20 items of the SRQ-20 in the last 4 weeks were considered as having mental distress, similar with the number of previous studies conducted in Ethiopia $(10,11)$. Trained data collectors and supervisors collected the data and supervised the data collection procedure respectively.

Data analysis: The data were entered into EpiInfo version 3.5.4 statistical software and analyzed using Statistical Package for Social Science (SPSS) version 20 statistical software. Descriptive statistics was computed for all variables according to their type. Frequency, mean and standard deviation was obtained for continuous variables while the categorical variables were assessed by computing frequencies and proportions. Multivariable analysis was performed following bivariate analysis to adjust for the effect of confounders using variables that have a $\mathrm{P}<0.25$. Both crude and adjusted odds ratio with a 95\% confidence interval were reported. Statistical significance was considered at a significance level of 5\%.

Ethical cconsideration: Ethical approval was obtained from the Institutional Ethical Review

DOI: http://dx.doi.org/10.4314/ejhs.v29i3.7 
Board of Hawassa College of Health Sciences. The nature of the study, the research objectives, and the confidentiality of the data were fully explained to the study subjects, and verbal informed consent for the study was taken from each participant. Data obtained from each study participant was kept confidential.

\section{RESULTS}

Two hundred sixty-four (126 male and 138 female) students participated in the study, with response rate of $94 \%$. The large majority of the study subjects $220(83.30 \%)$ were in the age group of 18-24 while more than one-third, 109(41.30\%) of the study subjects, were from Sidama ethnic group. Nearly two-thirds, 173(65.5\%), of the study subjects were Protestant while 195(73.90\%) and $212(80.30 \%)$ were single and third-year students respectively. Nearly one-third of the students, $77(29.20 \%)$, were from Midwifery Department. More than half, $139(52.75 \%)$, and $88(33.30 \%)$ of the study subjects were from urban areas and were living alone (Table 1). Regarding the habit of substance use, 43(16.30\%), 70(26.50\%) and $10(3.80 \%)$ chewing khat, using alcohol and other substances including cigarette/hashish, respectively (Table 2).

Using the SRQ-20 with the cutoff point of eight, the prevalence of mental distress among the study subjects was found to be $59(22.30 \%)$ with the mean \pm SD score of $4.87 \pm 3.45$. The prevalence of mental distress was relatively high, 39(28.3\%), among female college students as compared to the male students which were found to be $20(15.9 \%)$. As per the findings of multivariable logistic regression analysis conducted using significant variables in the bivariate analysis, the most important covariates identified were sex, year of study, ethnicity, and GPA. Female students were 2.08 times more likely to have mental distress as compared to male students $(\mathrm{AOR}=2.08$ [95\% CI: 1.14-3.82]). The odds of mental distress among third year students was $60 \%$ lower compared to second year students $[\mathrm{AOR}=$ $0.40 ; 95 \%$ CI, (0.19-0.87)]. Students who were from Amhara ethnic group were 3.37 times more likely to have mental distress compared to students from Sidama ethnic group $[\mathrm{AOR}=3.37$; 95\% CI (1.1310.09)].

Moreover, the GPAs of the students were statistically associated with mental distress where students with GPA of below $60 \%$ were 4.69 times more likely to be mentally distressed compared to students having GPAs of greater or equal to $60 \%$ $(\mathrm{AOR}=4.69 ; 95 \% \mathrm{CI},[2.46-8.96])$ (Table 3). 
Table 1: Demographic and Socio-economic Characteristics of Students, Hawassa City, 2015 (n=264).

\begin{tabular}{|c|c|c|c|}
\hline Variables & & Frequency & Percent \\
\hline \multirow[t]{2}{*}{ Sex } & Male & 126 & 47.70 \\
\hline & Female & 138 & 52.30 \\
\hline \multirow[t]{3}{*}{ Age groups } & $<18$ & 16 & 6.10 \\
\hline & $18-24$ & 220 & 83.30 \\
\hline & $>24$ & 28 & 10.60 \\
\hline \multirow[t]{6}{*}{ Ethnicity } & Sidama & 109 & 41.30 \\
\hline & Wolayta & 65 & 24.60 \\
\hline & Amhara & 39 & 14.80 \\
\hline & Gurage & 24 & 9.10 \\
\hline & Oromo & 16 & 6.00 \\
\hline & Others & 11 & 4.20 \\
\hline \multirow[t]{4}{*}{ Religion } & Protestant & 173 & 65.50 \\
\hline & Orthodox & 56 & 21.20 \\
\hline & Muslim & 23 & 8.70 \\
\hline & Other & 12 & 4.60 \\
\hline \multirow{3}{*}{ Marital status } & Single & 195 & 73.90 \\
\hline & Married & 65 & 24.60 \\
\hline & Other & 4 & 1.50 \\
\hline \multirow[t]{2}{*}{ Year of study } & Third year & 212 & 80.30 \\
\hline & Second year & 52 & 19.70 \\
\hline \multirow[t]{8}{*}{ Department } & Midwife & 77 & 29.20 \\
\hline & Clinical & 57 & 21.60 \\
\hline & HIT & 40 & 15.10 \\
\hline & Pharmacy & 21 & 8.00 \\
\hline & Public & 21 & 8.00 \\
\hline & Radiography & 21 & 8.00 \\
\hline & Laboratory & 19 & 7.20 \\
\hline & Anesthesia & 8 & 3.00 \\
\hline \multirow[t]{2}{*}{ Family place of residence } & Urban & 139 & 52.75 \\
\hline & Rural & 125 & 47.35 \\
\hline \multirow[t]{4}{*}{ Living condition } & Alone & 88 & 33.30 \\
\hline & Friend & 74 & 28.00 \\
\hline & Father \& mother & 60 & 22.70 \\
\hline & Other & 42 & 16.00 \\
\hline \multirow[t]{2}{*}{ Monthly personal income } & $<300$ & 217 & 82.20 \\
\hline & $>300$ & 47 & 17.80 \\
\hline \multirow[t]{2}{*}{ Academic performance } & $>=60$ & 60 & 22.70 \\
\hline & $<60$ & 204 & 77.30 \\
\hline
\end{tabular}

DOI: http://dx.doi.org/10.4314/ejhs.v29i3.7 
Table 1: The Habit of Substance Use among Students, Hawassa City, 2015 ( $\mathrm{n}=264)$.

\begin{tabular}{llll}
\hline Variables & & Frequency & Percent \\
\hline Khat Chewing & Users & 43 & 16.30 \\
& Non users & 221 & 83.70 \\
Drinking Alcohol & Users & 70 & 26.50 \\
& Non users & 194 & 73.50 \\
Other Substance Use & Users & 10 & 3.80 \\
(Cigarette, Hashish ) & Non users & 154 & 96.20 \\
\hline
\end{tabular}

Table 3: Bivariate and Multivariable Logistic Regression Analysis for Selected Factors Associated with Mental Mistress among Students, Hawassa City, 2015 ( $n=264)$.

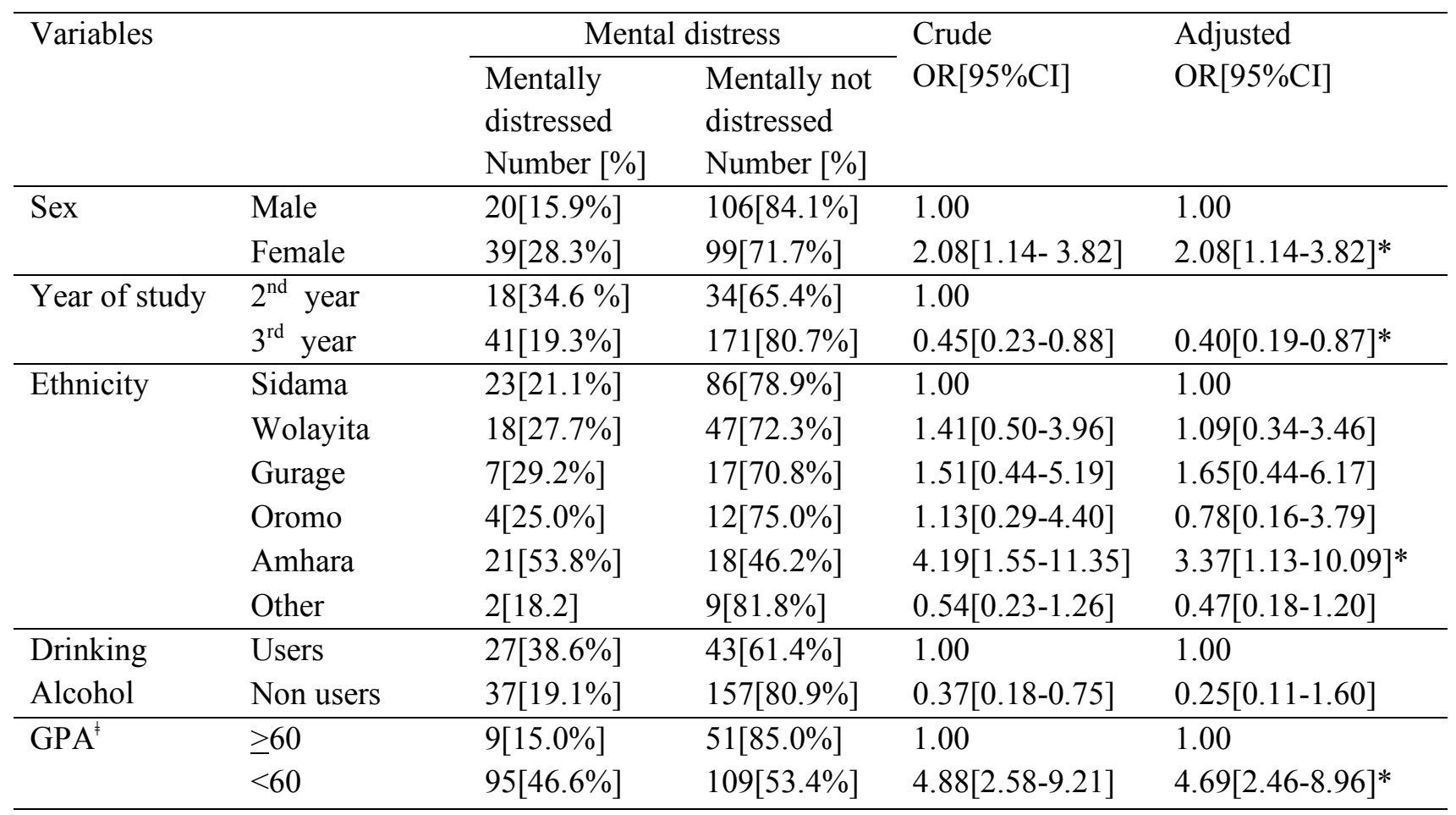

*indicate statistical significance: $\neq$-Grade Point Average

\section{DISCUSSION}

This study assessed the prevalence of mental distress and the associated risk factors among college students in Southern Ethiopia. The study showed that $59(22.3 \%)$ of the study subjects had mental distress based on the cutoff point of eight for SRQ-20. Studies conducted globally on mental distress among college students reported prevalence ranging from $17.70 \%$ to $41.1 \%(5-7)$.
Studies conducted in Ethiopia among students of higher academic institutions also reported similar variations from $21.6 \%$ to $49.1 \%(8-12)$. The use of varying cutoff points for determining mental distress because of the lack of a generally accepted cutoff score for the global community may be among the reasons for the observed variation in the reported prevalence (14). The variations related to the study settings, and socio-cultural and 
environmental factors might further contributed to the observed differences (11).

Female students were more likely to have mental distress compared to male students. Our finding was consistent with most of the reviewed literature from around the globe that documented that female students' experience mental distress more often compared to male students (4$7,9,10)$. The difference that female students experience more mental health problem than male students may be related to their difference in exposure to biological and environmental risk factors including ineffective coping mechanisms, expressing about their feelings and hormonal changes during their menstruation period $(6,17)$. Nonetheless, few others studies have reported no difference in the prevalence of mental distress between male and female students $(8,11,12)$.

The odds of mental distress among third-year students were lower compared to those of secondyear students. Our finding was in line with similar studies conducted in Hawassa, Ethiopia (11) and Punjab, India (5) which reported that as the year of study increases, the probability of having distress decreases. This may be explained by the fact that first-year students may face adjustment difficulty in relation to the new environment, new academic challenges, and separation from close family and old friends. The requirement for new social interaction with other students having mixed culture and separation from pre-existing family/social support could also have their own contribution for the observed higher level of mental distress among students in the earlier years of study $(10,11)$.

The risk of mental distress was more among students who had lower average GPAs than those who had higher GPAs. A similar finding was reported from a study conducted in Northwest part of Ethiopia (10). The possible explanation could be that students with lower GPA could not sleep, eat and relax because of their fear of failure, which may end up with academic dismissal. These stressful situations may eventually lead to mental health problem. There was also a significant association between mental distress and ethnicity. This could be because of differences in culture, religious practices and habits, which could contribute to mental distress.
The limitations of this study includes the fact that the study subjects were recruited from a single public college working under the Southern Regional Health Bureau, and some other relevant information such as family and social support was not collected. Furthermore, establishing a causal relationship, for example, ascertaining whether low GPAs lead to mental distress or that mental distress caused poorer performance in academics was found to be difficult due to the cross-sectional nature of the study. The use of a screening tool with no accepted cut-off score should also be considered as the limitation of the study.

In conclusion, this study revealed that the overall prevalence of mental distress among Hawassa College of Health Science students was 22.3\%. Sex, academic level, Ethnicity, and GPA were significantly associated with the likelihood of having mental distress. The finding suggests the importance of providing mental health counseling support for students as part of the teaching and learning process. Further research should be undertaken to examine multiple factors influencing the students' mental health condition.

\section{REFERENCES}

1. WHO. The WHO report 2001; Mental health: new understanding, new hope. Geneva, Switzerland; 2001.

2. WHO. Investing in mental health: Evidence for action. Geneva, Switzerland; 2003.

3. FDRE MOH. National Mental Health Strategy 2012/13 - 2015/16. Addis Ababa; 2015.

4. Kumar H, Shaheen A, Rasool I, Shafi M. Psychological Distress and Life Satisfaction among University Students. J Psychol Clin Psychiatry. 2016;5(3): 00283-9.

5. Mehta K, Kaur S, Girgla KK, Kaur P, Kaur H. A study of mental distress in medical students. Natl J Physiol Pharm Pharmacol 2015;5190194. 2015;5(3):190-4.

6. Hersi L, Tesfay K, Gesesew H, Krahl W, Ereg D, Tesfaye M. Mental distress and associated factors among undergraduate students at the University of Hargeisa, Somaliland : a cross sectional study. Int $J$ Ment Health Syst. 2017;11:39.

7. Poorolajal J, Ghaleiha A, Darvishi N, Daryaei

DOI: http://dx.doi.org/10.4314/ejhs.v29i3.7 
S. The Prevalence of Psychiatric Distress and Associated Risk Factors among College Students Using GHQ-28 Questionnaire. Iran J Public Heal [Internet]. 2017;46(7):957-63.

8. Yadeta D, Jemal E, Tadesse A. Mental distress among university students in Ethiopia: a cross sectional survey. Pan Afr Med $J$. 2013;15:95.

9. Tariku GH, Zerihun A, Bisrat Z, Jini D. Mental Distress and its Associated Factors among Students of Mizan Aman Health Science College, Ethiopia. J Med Sci. 2017;17(2):61.

10. Dachew BA, Bisetegn TA, Gebremariam RB. Prevalence of Mental Distress and Associated Factors among Undergraduate Students of University of Gondar, Northwest Ethiopia: A Cross-Sectional Institutional Based Study. PLoS One. 2015;10(3):1-10.

11. Melese B, Bayu B, Wondwossen F, Tilahun $\mathrm{K}$, Lema S, Ayehu M, et al. Prevalence of mental distress and associated factors among Hawassa University medical students , Southern Ethiopia: a cross - sectional study. BMC Res Notes. 2016;9:485.

12. Tesfaye A. Prevalence and correlates of mental distress among regular undergraduate students of Hawassa University: a cross sectional. East Afr $J$ Public Heal. 2009;6(1):85-94.

13. FDRE-CSA. Federal Democratic Republic of Ethiopia Central Statistical Agency Population Projection of Ethiopia for All Regions At Wereda Level from 2014 - 2017. 2013; (August 2013).

14. M. Beusenberg and J.Orley. A User's guide to the self reporting questionnaire (SRQ). WHO. Division of Mental Health; 1994.

15. Hunduma G, Girma M, Digaffe $T$, Weldegebreal F, Tola A. Prevalence and determinants of common mental illness among adult residents of Harari Regional State, Eastern Ethiopia. Pan Afr Med J. 2017;28:262.

16. Damena T, Mossie A, Tesfaye M. Khat chewing and mental distress: a community based study, in Jimma City, Southwestern Ethiopia. Ethiop J Heal Sci. 2011;21(1):3745.

17. Abrar A, Kazim M, Hanif M, Mansoor S, Tahir S. Prevalence of anxiety and depression among medical students of shifa college of medicine. Pakistan $J$ Neurol Sci. 2014;9(3):12-15 